\title{
Thermalization of hadrons via Hagedorn states
}

\author{
M. Beitel, K. Gallmeister, and C. Greiner \\ Institut für Theoretische Physik, Goethe-Universität Frankfurt
}

Hagedorn states are part of the Statistical Bootstrap model (SBM) [1] having remarkable features. They are very massive hadron-like resonances which are allowed to have any quantum numbers as long as they are compatible with their mass. Hence Hagedorn states can carry charges like baryon number, strangeness or electric charge exceeding those of known hadrons. To obtain the whole zoo of Hagedorn states we solve, in contrast to Ref. [2] where a non-covariant version was examined, a covariant bootstrap equation numerically by ensuring energy-momentum conservation and the conservation of the baryon number $B$, strangeness $S$, and electric charge $Q$. As basis for this bootstrap we use the mass distributions of the known hadronic resonances, as implemented in the transport code UrQMD. The solution provides Hagedorn spectra for different quantum number combinations as function of Hagedorn state's mass. The spectra for charge neutral, mesonlike, $(B=S=Q=0)$ Hagedorn states for two different Hagedorn state radii are shown in Fig. 1. The sum of the hadronic spectral functions with the same quantum numbers is presented to emphasize that our Hagedorn spectra not only exhibit the exponential behaviour for very large Hagedorn state masses but also reproduce the low-mass part. Using these spectra one can formulate the decay widths $\Gamma$ of Hagedorn states needed in cascading simulations. The decay width of charge neutral Hagedorn states is presented in Fig. 2. As an important feature, all decay widths tend to a constant value greater zero in the infinite mass limit denoting that all Hagedorn states must be unstable.

Now having the decay widths we proceed to examine a decay of a massive Hagedorn state in a cascade until stable hadrons are left only. Apart from the calculated hadronic multiplicities we also looked at the energy distribution of those final particles. We found that all particles exhibit the same temperature, obtained from the Boltzmann distribution's slope as can be seen in Fig. 3. Another remarkable feature is that this (thermodynamic) temperature exactly equals the Hagedorn temperature you obtain from the slopes of the Hagedorn spectra shown in Fig. 1. A detailed discussion about these findings can be found in [3].

\section{References}

[1] R. Hagedorn, Nuovo Cim. Suppl. 3, 147 (1965).

[2] C. J. Hamer and S. C. Frautschi, Phys. Rev. D 4, 2125 (1971) [Erratum-ibid. D 5, 1235 (1972)].

[3] M. Beitel, K. Gallmeister and C. Greiner, Phys. Rev. C 90, 045203 (2014) [arXiv:1402.1458 [hep-ph]].

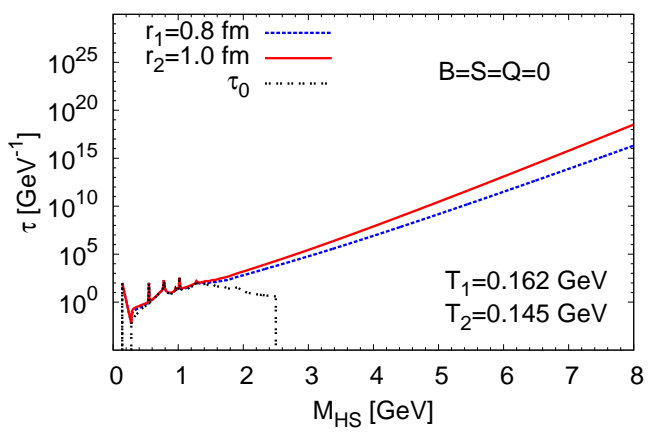

Figure 1: Meson-like $(B, S, Q=0)$ Hagedorn spectra.

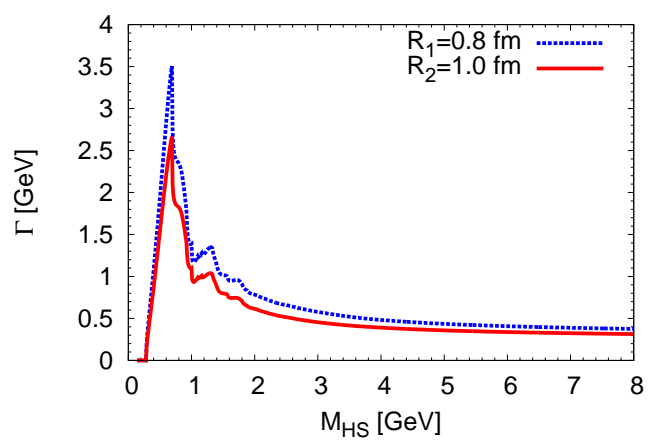

Figure 2: Decay widths of Hagedorn states as in Fig. 1.

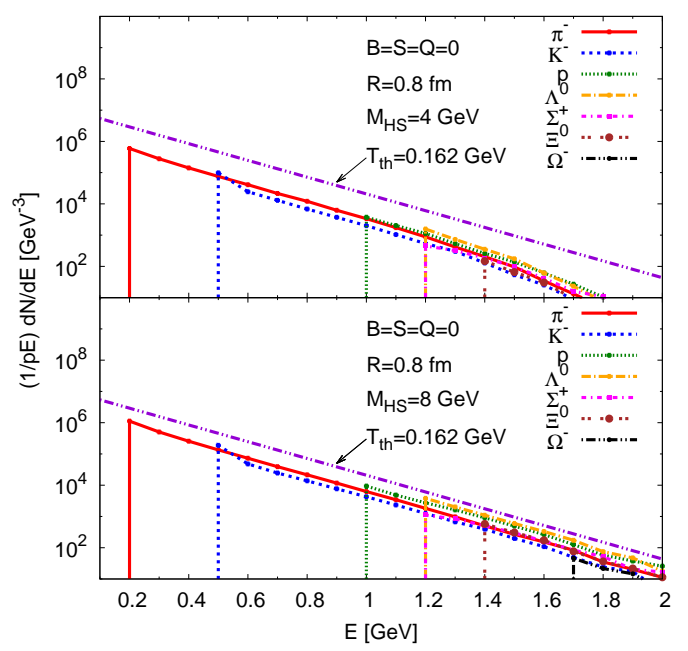

Figure 3: Energy distribution of final hadrons stemming from an initial charge neutral $(B, S, Q=0)$ Hagedorn state with $r=$ $0.8 \mathrm{fm}$ for two initial masses $M_{\mathrm{HS}}=4 \mathrm{GeV}$ (up) and $M_{\mathrm{HS}}=$ $8 \mathrm{GeV}$ (down) 